\title{
Mirroring the emotions of others by autonomic system: intra-species effect in children
}

\author{
Maria E. Vanutelli ${ }^{1,2,3}$ - Irene Venturella ${ }^{1,2}$ \\ Laura Angioletti ${ }^{1}$ - Michela Balconi ${ }^{1,2}$ \\ ${ }^{1}$ Department of Psychology, Catholic University of the Sacred Heart, Milan, Italy \\ ${ }^{2}$ Research Unit in Affective and Social Neuroscience, Catholic University of the Sacred \\ Heart, Milan, Italy \\ ${ }^{3}$ Department of Philosophy, University of Milan, Italy
}

DOI: http://dx.doi.org/10.7358/neur-2017-022-vanuｍaria.vanutelli@unimi.it

\section{ABSTRACT}

Previous research investigated the presence of differential autonomic responses towards the emotions expressed by individuals from ingroup or outgroup contexts. Results found increased affective reactions and typical identification bias that can be explained, according to "the similarity factor", as the tendency to tune stronger with those perceived as more similar. Then, few previous studies explored the presence of such mechanisms in intra and interspecies contexts. However, further investigation is needed to better explore these issues in developmental samples and to different emotionally valenced stimuli. Thus, we asked 30 children to watch emotional pictures involving child-child (CC) and child-animal (CA) interactions with negative, positive, or neutral valence. Autonomic responses were recorded and compared across species and gender. Results showed a higher sensitivity to intraspecies emotional valence (increased heart rate for negative compared to neutral stimuli) which was visible only in the female group, thus confirming the presence of a gender effect.

Keywords: Intralinterspecies interactions; Emotion perception; Similarity factor; Autonomic measures; HR; EDA 


\section{INTRODUCTION}

Emotions have been recognized as the causes, mediators, and consequences of our social interactions and they are considered as fundamental in our everyday lives. Also, they can be observed as ongoing transformations within the relationship between two or more agents inserted in a social context (de Rivera \& Grinkis, 1986), being able to improve the ability of individuals to adapt in some specific situations.

For these reasons, recent research in the field of social and affective neuroscience has increasingly investigated people emotional reactions to various affective social contexts by inducing emotional experience and, consequently, by observing typical psychophysiological responses. Indeed, it is possible to understand which situations are perceived as the most emotionally salient by measuring specific autonomic variations like cardiovascular indices or electrodermal activity or facial expressions (Balconi \& Bortolotti, 2012, 2014; Balconi, Falbo, \& Brambilla, 2009; Balconi, Grippa, \& Vanutelli, 2015; Balconi \& Pozzoli, 2008).

However, three main limitations can be found with regard to previous work: first, the presence of a confounding species effect in the use of emotional pictures. Secondly, the exclusive induction of negative emotions in those few studies considering both intra and interspecies contexts. Thirdly, the lack of insights on early phases of ontogenesis.

For what concerns the first point, in fact, previous studies used mixed affective pictures taken from standardized databases involving both human and animals' scenes in positive and negative contexts. However, these different types of interactions in terms of species have different evolutionary and biological meaning for human subjects and so they could be distinguished for their characteristics, in order to explore similarities and specificities of these stimuli in inducing emotional responses. In fact, within the field of ethology and evolutionary psychology, Preston and de Waal (Preston \& de Waal, 2002) theorized the Perception-Action model of emotions (PAM). The model suggests that others' emotional states triggers automatic peripheral (and neural) responses of congruent states in the observer. Also, according to what they called the "similarity factor", we experience the tendency to tune stronger with those perceived as more similar based on some features such as personality characteristics (Gruen \& Mendelsohn, 1986), appearance (Brown, Bradley, \& Lang, 2006), cultural similarity, or social circumstances (Westbury \& Neumann, 2008). Starting from this evidence, some studies explored the presence of ingroup/outgroup identification bias (see for example Brown et al., 2006 or Forgiarini, Gallucci, \& Maravita, 2011).

Nonetheless, the originality of this line of research lies in the idea to compare human and animal actors as sources of different emotional sharing and tuning. For example, Westbury and Neumann (2008) hypothesized that a linear pattern of responses would have been found across increasing phylogenetic 
relatedness, with respect to the similarity hypothesis. They asked participants to watch ecological real-life film stimuli depicting humans, primates, quadruped mammals and birds in victimized circumstances while electromyogram (EMG) and skin conductance response (SCR) were recorded. Results confirmed their predictions and in particular SCR decreased as a function of phylogenetic distance. Also, Prguda and Neumann (2014) presented their participants with images of infants and adults from both human and wild non-human species (primates, mammals, and birds) depicted in dangerous negative situations during autonomic activity recording. Results showed stronger autonomic responses and higher subjective empathy ratings for human scenes, especially with infant subjects, but the effect did not extend to non-human scenarios.

Secondly, these few studies mainly focused on empathic mechanisms elicited by negative, aversive conditions and, thirdly, they only involved adult participants, while less attention was given to developmental samples. However, it could be important to associate and distinguish different emotional scenarios among intra and interspecies contexts, to explore if and how they can influence affective responses with specific and typical patterns. Also, it could be interesting to investigate the similarity factor also at earlier stages of life.

To answer these questions, in the present study a sample of children was recruited and asked to passively view emotional pictures depicting both childchild (CC) and child-animal (CA) interactions with negative, positive, or neutral valence while their cardiovascular indices (heart rate: HR) and electrodermal activity (skin conductance level and response: SCL, SCR) were recorded. Also, children's gender was considered as a possible significant factor in modulating emotional responses. In fact, many previous studies have reported consistent evidence of sex differences in emotional reactivity to affective pictures, with women generally showing stronger physiological reactions to especially negative, aversive pictures (Althaus et al., 2014) and more distress to fearful and stressful experiences (Kudielka, Buske-Kirschbaum, Hellhammer, \& Kirschbaum, 2004). Such effects have also been replicated in laboratory ecological stress-related tasks. For example, Heponiemi, KeltikangasJärvinen, Kettunen, Puttonen, and Ravaja (2004) and Ditzen et al. (2007) found increased heart rate in women subjects during stressful, negative conditions within psychosocial stress conditions, involving for example speech tasks or mental arithmetic tests in front of an audience.

According to the similarity factor, we hypothesized a species effect due to the presence of an identification bias with intraspecies emotional situations. Based on previous research, we predicted that such effect could be visible in the form of increased autonomic responses to contexts involving human interactions, with respect to both electrodermal and cardiovascular indices (higher peripheral activation: quantitative effect). Accordingly, we also expected that intraspecies emotional contexts could benefit of more refined activation patterns based on 
valence factor, that is specific autonomic responses to positive, negative, or neutral pictures (differential peripheral activation: qualitative effect). Moreover, we predicted that the highest responses could be elicited by negative, aversive pictures, because of enhanced emotional and attentional processes. Finally, a gender effect was hypothesized, with female children being more sensitive than males to highly emotional pictures in terms of autonomic activation.

\section{MATERIALS AND METHODS}

\subsection{Participants}

A sample of 30 children, 15 females and 15 males $\left(\mathrm{M}_{\text {age }}=7.7 ; \mathrm{SD}=1.83\right.$; range 3-11) took part in the present experiment. Children's caregivers affirmed that they were healthy, with normal or corrected-to-normal visual acuity. Informed consent was obtained from the legal guardian of each child, which also provided verbal assent to participate in the study. Children reported to be familiar with cats and dogs (the animal actors depicted in the emotional scenes; see next paragraph). Exclusion criteria were neurological or psychiatric pathologies. The research was approved by the ethical committee of the institution (Department of Psychology, Catholic University of Milan) where the work was carried out.

\subsection{Stimuli}

Affective pictures depicting child-child (CC) and child-animal (CA) interactions were displayed to the participants. The stimuli set was constituted by 48 coloured realistic images representing positive, negative and neutral interactions between children (24 pictures) and children with animals (24 pictures). Pictures were equally divided in three different categories with respect to emotional valence (positive, negative and neutral scenes) (see Fig. 1). All of them were controlled for size $(14 \mathrm{~cm} \times 10 \mathrm{~cm})$ and they were similar for perceptual features such as luminance and complexity (i.e. number of details in the scene). Moreover, scenes were also balanced with respect to actors' sex and animals' species (dogs and cats). Each stimulus was presented three times for a total of 144 stimulations, subdivided in 6 blocks of 24 pictures. This experimental set of stimuli was created by replicating those used in a previous experiment with adults subjects and adults actors (see Balconi \& Vanutelli, 2015, 2016; Vanutelli \& Balconi, 2015). 


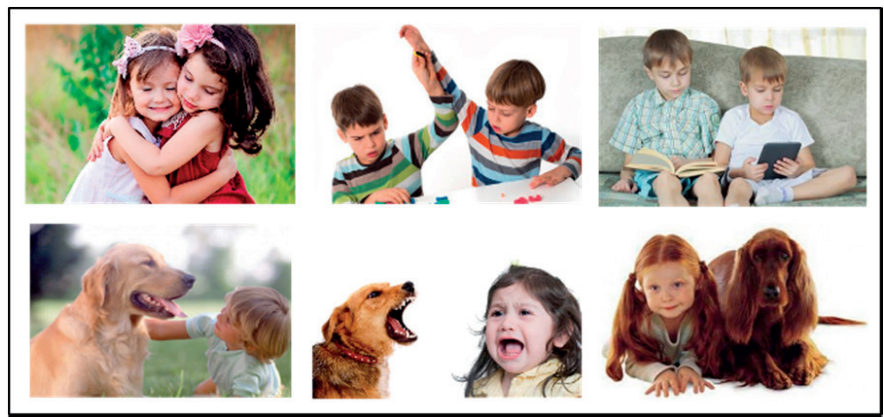

Figure 1. Examples of child-child (CC: top) and child-animal (CA: bottom) interactions with positive (left), negative (center) and neutral (right) valence

\section{PROCEDURE}

We administered the experimental phase in a soft lighted and sound isolated room, and children were seated on a chair in front of a computer monitor (distance: $70 \mathrm{~cm}$ ). The set of stimuli was presented using E-Prime 2.0 software (Psychology Software Tools Inc., Sharpsburg, PA, USA) running on a personal computer with a 15-inch screen. Participants were asked to observe each stimulus during autonomic measure recording, and to watch the images for the entire time of exposure. In order to facilitate empathic resonance with the reproduced scene, actors represented in the pictures were similar in age to the experimental participants. Pictures were randomly presented at the centre of the computer monitor for 6 seconds, with an inter-stimulus interval of 8 seconds. Each picture was presented for three times $(24$ stimuli for each category), for a total of 72 presentations for both the CC and the CA condition. 120 seconds resting baseline was recorded at the beginning of the experiment. An experimenter supported children in keeping their attention focused on the set of stimuli: she intervened in case of children's difficulty during the progression of the task.

\subsection{Autonomic measures recording and analysis}

For the present study Biofeedback 2000 ${ }^{\mathrm{x}-\mathrm{pert}}$ system with radio module MULTI (Schuhfried GmbH, Mödling, Austria) was used, which is capable of measuring skin conductance level and response (SCL, SCR; in $\mu$ S) and heart rate (HR; in beats per minute, bpm). SCL was recorded with an EDA1 gold electrode using current-voltage measurement at a sampling rate of $2 \mathrm{kHz}$. The use of alternating voltage prevents polarization. The measurement resolution for the 
SCL measurement is $1 \mathrm{nS}$. SCR was recorded with a sampling rate of $40 \mathrm{~Hz}$. HR was measured by infrared absorption principle with a sampling rate of 500 $\mathrm{Hz}$. Measurement resolution is $.004 \mathrm{bpm}$.

Moreover, the mobility of the non-dominant hand was monitored with an accelerometer in $\mathrm{m} / \mathrm{s} 2$ integrated into the sender unit to ensure that recordings were not compromised by hand movements. All sensors were combined in one unit which was attached to the volar surface of the distal section of the forefinger of the non-dominant hand.

\section{RESULTS}

SCL, SCR and HR measures were analyzed with three separated two factor (2 species $\mathrm{x} 3$ valence) repeated measure factors. Since no statistically significant results emerged for electrodermal indices (nor SCL, nor SCR), only results for HR measure will be disclosed. A significant Species $x$ Valence interaction effect was found $\left(\mathrm{F}_{2,56}=55.1 ; \mathrm{p}<0.005 ; \eta^{2}=0.2\right)$. Post-hoc comparisons showed that negative CC stimuli induced a significant $(\mathrm{p}<0.05)$ increase in HR response $(\mathrm{M}=84.9, \mathrm{SD}=2.47)$ than neutral ones $(\mathrm{M}=82.23, \mathrm{SD}=2.33)$. Conversely, no significant difference emerged between negative and positive pictures, or between positive and neutral.

In addition, a significant Species $\mathrm{x}$ Valence $\mathrm{x}$ Gender interaction effect was found $\left(\mathrm{F}_{2,56}=45.95 ; \mathrm{p}<0.005 ; \eta^{2}=0.17\right)$ revealing that negative $\mathrm{CC}$ stimuli inducing significantly $(\mathrm{p}<0.05)$ higher $\mathrm{HR}$ responses $(\mathrm{M}=85.35, \mathrm{SD}$ $=3.5)$ than neutral ones $(\mathrm{M}=81.14, \mathrm{SD}=3.3)$ in females. Conversely, such effect was not present in the males group (see Fig. 2). 

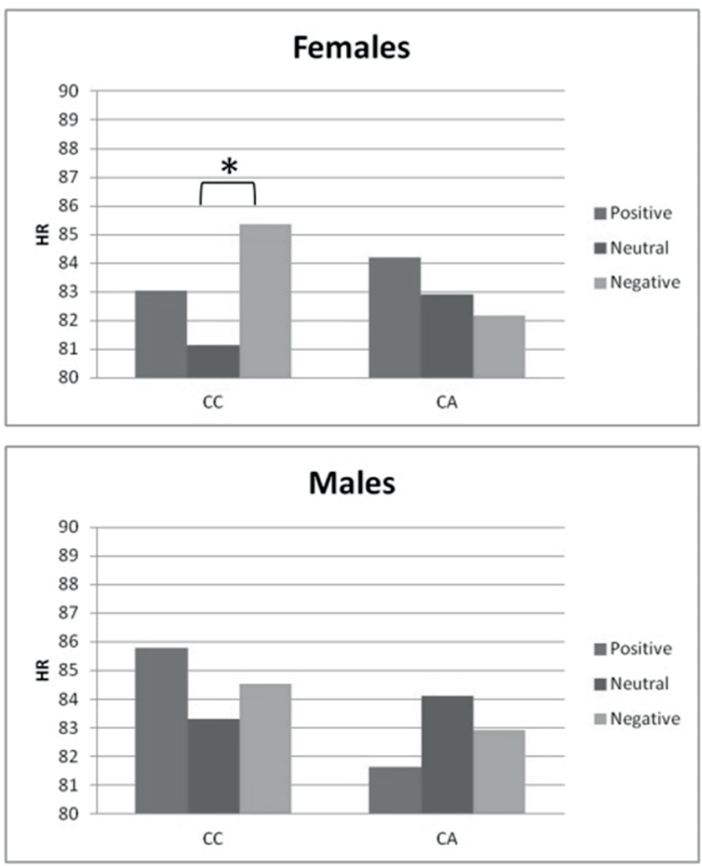

Figure 2. HR responses as a function of species (intra vs inter) and children's gender (females: top; males: bottom). The data show a species $x$ valence effect within the female group for intraspecies contexts (CC)

\section{DISCUSSION}

Because of the great importance attributed to emotions in social contexts, previous neuroscientific research investigated people emotional reactions by inducing emotional experience with various affective pictures, to observe and measure typical psychophysiological responses (Balconi \& Bortolotti, 2012, 2014; Balconi et al., 2015). However, previous studies did not consider some specific variables of interest to emotional experience: first, they used mixed affective pictures taken from standardized databases involving both humans and animals scenes in positive and negative contexts, even if the two categories assume different evolutionary and biological meaning for human subjects. Also, those few previous studies which distinguished the two categories mainly focused on empathy for pain and negative conditions (second point) and to adult samples (third point). Thus, the present study aimed at exploring the 
differential contributions of these factors in children with an emotional paradigm involving both child-child (intraspecies) and child-animals (interspecies) interactions in positive, negative, or neutral contexts.

Results showed an interaction effect between species and valence factors, with increased heart rate to aversive situations with respect to neutral ones, but only during CC interactions. This result could be interpreted at light of the similarity factor: in fact, thanks to a better knowledge of the emotional experience and expression involving human subjects, stimuli affective valence produced a differential effect in autonomic responses (qualitative effect), while a similar effect was not present in CA situations. In fact, it is possible to assume that, within CC domain, more refined emotional abilities could have led children to better perceive context's valence between peers, that is also disapproved and discouraged from significant adults (like parents at home, or teachers at school).

Indeed, for what concerns valence modulation, such result highlights that scenes depicting quarrels between children are perceived as the most orienting and stressful, as displayed by increased HR response. Such effect is in line with previous research reporting the presence of a negativity bias towards aversive conditions, in both computerized tasks (Balconi et al., 2015) and ecological situations (Kudielka et al., 2004).

Finally, according to gender effects found both in adults (Ditzen et al., 2007; Heponiemi et al., 2004; Kudielka et al., 2004) and children (Kudielka et al., 2004; McManis, Bradley, Berg, Cuthbert, \& Lang, 2001), these findings were present only in the female group, offering further support to the notion that female subjects are more reactive to highly aversive cues. In fact, they showed more differentiation in HR activity when viewing negative scenarios, with respect to neutral ones, while this effect was not visible for males.

However, contrary to our expectations, a main effect of species with increased autonomic responses to $\mathrm{CC}$ with respect to $\mathrm{CA}$ (quantitative effect) did not emerge. Maybe, a distinction between intra and interspecies contexts is not sufficiently clear to show significant quantitative effects in children. In fact, it is possible to assume that, during time, the similarity factor becomes stronger and more supported by different experiences, as well as by social and cultural issues.

Also, it was possible to identify significant results only for HR, and not for electrodermal indices. The lack of significant differences could be ascribed to the type of information that these different measures can provide: in fact, it has been shown that $\mathrm{HR}$ is especially sensitive to the valence dimension of emotional response (Lang, 1995) and can be a rich source of information concerning attentional and cognitive processing (Sohn, Sokhadze, \& Watanuki, 2001), while the magnitude of electrodermal activity can be used as an indicator of the quantitative aspects of emotional arousal (Sohn et al., 2001). Thus, according to what previously discussed, it is possible to hypothesize the presence of a significant qualitative effect in terms of valence, 
shown by HR increase to negative pictures, together with the absence of a clear quantitative effect in terms of arousal. Nonetheless, these points should be better explored in future research.

To conclude, the present study provides initial evidence about autonomic response in children during affective stimulation involving both intra and interspecies interactive contexts. The similarity factor induced an autonomic activity modulation to intragroup interactions (identification bias), while negative contexts clearly induced higher responses according to the negativity bias. Also, gender proved to be a meaningful factor to consider, since the effect was present only in female children.

Nevertheless, future research could deepen these issues by directly comparing, for example, different developmental ages until adulthood to investigate if (and how) the affective responses related to the similarity factor change in time. Also, supplementary neuroscientific measures such as electroencephalography (EEG) or other neural indices could be applied, to explore the relation between peripheral and neural responses. Finally, explicit and subjective measures could be considered, to explore the interplay between covert and overt responses, as well as the role of individual factors, such as the presence of siblings and pets in the household, but also personality components, including empathic and motivational factors.

\section{REFERENCES}

Althaus, M., Groen, Y., Van Der Schaft, L., Minderaa, R. B., Tucha, O., Mulder, L. J. M., \& Wijers, A. A. (2014). Sex differences in orienting to pictures with and without humans: Evidence from the cardiac evoked response (ECR) and the cortical long latency parietal positivity (LPP). PLoS ONE, 9(10).

Balconi, M., \& Pozzoli, U. (2008). Event-related oscillations (ERO) and eventrelated potentials (ERP) in emotional face recognition. International Journal of Neuroscience, 118(10), 1412-1424.

Balconi, M., Falbo, L., \& Brambilla, E. (2009). BIS/BAS responses to emotional cues: Self report, autonomic measure and alpha band modulation. Personality and Individual Differences, 47(8), 858-863.

Balconi, M., \& Bortolotti, A. (2012). Resonance mechanism in empathic behavior. BEES, BIS/BAS and psychophysiological contribution. Physiology and Behavior, 105(2), 298-304.

Balconi, M., \& Bortolotti, A. (2014). Self-report, personality and autonomic system modulation in response to empathic conflictual versus non conflictual situation. Cognition \& Emotion, 28(1), 153-162. 
Balconi, M., Grippa, E., \& Vanutelli, M. E. (2015). What hemodynamic (fNIRS), electrophysiological (EEG) and autonomic integrated measures can tell us about emotional processing. Brain and Cognition, 95, 67-76.

Balconi, M., \& Vanutelli, M. E. (2015). Emotions and BIS/BAS components affect brain activity (ERPs and fNIRS) in observing intra-species and inter-species interactions. Brain Imaging and Behavior, 10(3), 750-760.

Balconi, M., \& Vanutelli, M. E. (2016). Hemodynamic (fNIRS) and EEG (N200) correlates of emotional inter-species interactions modulated by visual and auditory stimulation. Scientific Reports, 6 .

Brown, L. M., Bradley, M. M., \& Lang, P. J. (2006). Affective reactions to pictures of ingroup and outgroup members. Biological Psychology, 71(3), 303-311.

de Rivera, J., \& Grinkis, C. (1986). Emotions as social relationships. Motivation and emotion, 10(4), 351-369.

Ditzen, B., Neumann, I. D., Bodenmann, G., von Dawans, B., Turner, R. A., Ehlert, U., \& Heinrichs, M. (2007). Effects of different kinds of couple interaction on cortisol and heart rate responses to stress in women. Psychoneuroendocrinology, 32(5), 565-574.

Forgiarini, M., Gallucci, M., \& Maravita, A. (2011). Racism and the Empathy for Pain on Our Skin. Frontiers in Psychology, 2, 108.

Gruen, R. J., \& Mendelsohn, G. (1986). Emotional responses to affective displays in others: The distinction between empathy and sympathy. Journal of Personality and Social Psychology, 51(3), 609.

Heponiemi, T., Keltikangas-Järvinen, L., Kettunen, J., Puttonen, S., \& Ravaja, N. (2004). BIS-BAS sensitivity and cardiac autonomic stress profiles. Psychophysiology, 41(1), 37-45.

Kudielka, B. M., Buske-Kirschbaum, A., Hellhammer, D. H., \& Kirschbaum, C. (2004). Differential heart rate reactivity and recovery after psychosocial stress (TSST) in healthy children, younger adults, and elderly adults: The impact of age and gender. International Journal of Behavioral Medicine, 11(2), 116-121.

Lang, P. J. (1995). The emotion probe: Studies of motivation and attention. American Psychologist, 50(5), 372-385.

McManis, M. H., Bradley, M. M., Berg, W. K., Cuthbert, B. N., \& Lang, P. J. (2001). Emotional reactions in children: verbal, physiological, and behavioral responses to affective pictures. Psychophysiology, 38(2), 222-231.

Preston, S. D., \& de Waal, F. B. M. (2002). Empathy: its ultimate and proximate bases. The Behavioral and Brain Sciences, 25(1), 1-20. 
Prguda, E., \& Neumann, D. L. (2014). Inter-human and animal-directed empathy: a test for evolutionary biases in empathetic responding. Behavioural Processes, 108, 80-86.

Sohn, J. H., Sokhadze, E., \& Watanuki, S. (2001). Electrodermal and cardiovascular manifestations of emotions in children. Journal of Physiological Anthropology and Applied Human Science, 20(2), 55-64.

Vanutelli, M. E., \& Balconi, M. (2015). Perceiving emotions in human-human and human-animal interactions: Hemodynamic prefrontal activity (fNIRS) and empathic concern. Neuroscience Letters, 605, 1-6.

Westbury, H. R., \& Neumann, D. L. (2008). Empathy-related responses to moving film stimuli depicting human and non-human animal targets in negative circumstances. Biological Psychology, 78(1), 66-74. 
